


\section{Notice}

This report was prepared as an account of work sponsored by an agency of the United States government. Neither the United States government nor any agency thereof, nor any of their employees, makes any warranty, express or implied, or assumes any legal liability or responsibility for the accuracy, completeness, or usefulness of any information, apparatus, product, or process disclosed, or represents that its use would not infringe privately owned rights. Reference herein to any specific commercial product, process, or service by trade name, trademark, manufacturer, or otherwise does not necessarily constitute or imply its endorsement, recommendation, or favoring by the United States government or any agency thereof. The views and opinions of authors expressed herein do not necessarily state or reflect those of the United States government or any agency thereof. Subcontract number AAX-1-30482-01. Publication number NREL/SR-550-32390. Available electronically at http://www.osti.gov/bridge.

NREL is the U.S. Department of Energy's premier laboratory for renewable energy \& energy efficiency research, development, and deployment. 


\title{
Summary of Green Building Programs
}

\author{
Prepared for: \\ National Renewable Energy Laboratory \\ Golden, Colorado \\ Prepared by: \\ National Association of Home Builders (NAHB) Research Center, Inc. \\ Upper Marlboro, Maryland \\ Second Edition \\ August 2002
}




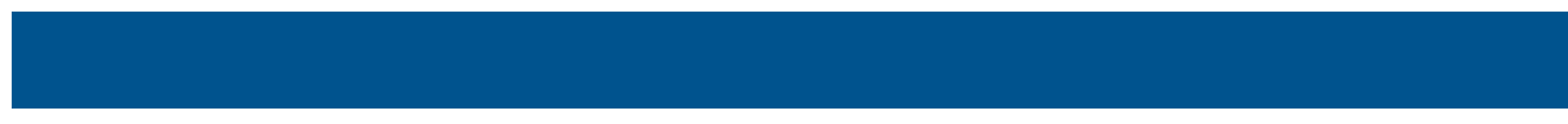




\section{Foreword}

In early 2002, the National Association of Home Builders (NAHB) Research Center completed a census of residential green building programs across the United States to assess differences and similarities among programs. Although the Research Center recognizes that other (e.g., commercial) green building programs exist, the focus of this report is on residential programs. In addition to presenting basic facts about the programs, the information provided catalogs different ways that builders participate in green building programs. This guide assumes that readers have a basic understanding of green building techniques.

\section{Key Findings}

Basic Facts

More than 18,000 homes have been built in compliance with the 26 green building programs surveyed in this study. Most of these homes are in Denver, Austin, and Seattle. The Austin and Denver programs have the most builder members; the Austin program has been in existence the longest.

\section{Most Established Programs}

Built Green Colorado in Denver and the Austin Energy Green Building Program in Texas are the largest and best established green building programs in the country. Built Green Colorado was established in 1995 and currently has 111 builders participating in the program. More than 9,000 homes have been completed to date in accordance to the program's guidelines. Participation in the Built Green Colorado program is voluntary. Builders receive marketing materials and recognition in the market.

Austin Energy's Green Building Program was established in 1990. This voluntary program currently has 111 builders participating, with more than 2,000 homes completed to date in accordance with the program's guidelines.

\section{Programs with Mandatory Participation}

For builders in Boulder, Colorado, and Frisco, Texas, compliance with the local green building program is required to get building permits.

\section{Programs in Development}

Green building programs in the early stages of development include Southern Arizona Green Building Alliance, Western North Carolina Green Building Council, Alameda County (California), Chula Vista (California), Hudson Valley Home Builders Association (HBA) Green Building Program (New York), and the Schenectady HBA Green Building Program (New York). 


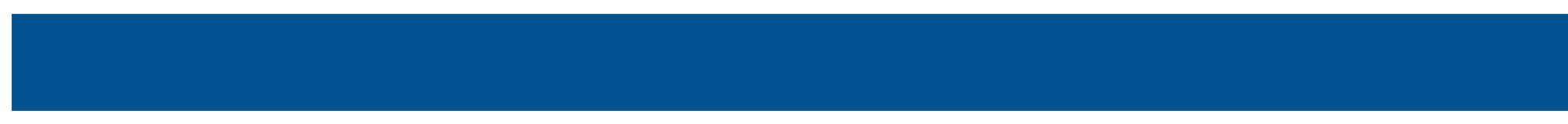




\section{Table of Contents}

1. Green Built Home (Wisconsin Environmental Initiative) .1

2. Build A Better Kitsap Home Builder Program (Kitsap HBA, Washington) .............. 3

3. EarthCraft House (Greater Atlanta HBA, Georgia) ............................................... 4

4. Built Green ${ }^{T M}$ Colorado (HBA of Metro Denver) ....................................................... 6

5. Built Green ${ }^{T M}$ (MBA of King and Snohomish Counties, Washington) ...................... 8

6. Green Home Designation (Florida Green Building Coalition) .............................. 10

7. City of Boulder Green Points (Colorado) ..................................................................... 12

8. Green Building Program, Austin Energy (Texas) ...................................................... 13

9. City of Scottsdale Green Building Program (Arizona) .......................................... 15

10. New Mexico Building America Partner Program (HBA of Central New Mexico)

11. County of Santa Barbara Innovative Building Review Program (California) .... 17

12. Build a Better Clark (Clark County, Washington HBA) .......................................... 18

13. Earth Advantage Program (Portland General Electric, Oregon) ........................ 20

14. G/Rated (City of Portland, Oregon) ...................................................................... 22

15. Home Builders Association of Greater Kansas City (Missouri) .............................. 24

16. City of Frisco (Texas) Green Building Program ........................................................ 25

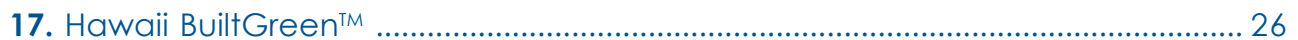

18. California Green Builder Program ......................................................................... 28

19. Green Built Program (HBA of Greater Grand Rapids, Michigan) ........................ 29

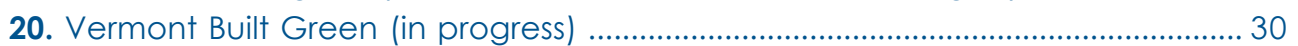

21. Southern Arizona Green Building Alliance (in progress) ........................................ 32

22. Western North Carolina Green Building Council (in progress) ............................. 32

23. Alameda County (California) (in progress) ............................................................ 33

24. Chula Vista (California) GreenStar Building Incentive Program (in progress) .. 33

25. Hudson Valley HBA Green Building Program (New York) (in progress) ................ 34

26. Schenectady HBA Green Building Program (New York) ..................................... 34 


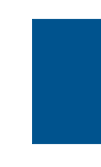




\section{Green Built Home (Wisconsin Environmental Initiative)}

\section{Overview:}

Checklist with very little weighting — the most points that are allocated to any one practice is four.

\section{Certification method:}

For each home, the builder submits a checklist, registration form, and a working set of plans. Green Built Home conducts an initial plan review and conducts random site visits.

\section{Levels of certification:}

One

\section{Qualification:}

Minimum of 50 points to qualify. Point breakdown is as follows: meet all mandatory requirements ( 15 points), and include a minimum of 35 additional points from any combination of other categories from the checklist (out of 232 total checklist points).

Year of inception:

$$
1999
$$

Number of builders:

$$
30
$$

Incentives offered to builders to participate:

Logos in homes, yard signs, plaques on certified homes, local press, ribbons and shirts for builders.

\section{Number of homes constructed to date:}

$$
202
$$

Contact information:

Green Built Home

Wisconsin Environmental Initiative

John Imes

16 N. Carroll Street, Suite 840

Madison, WI 53703-2726

p. $608-280-0360$

f. 608-280-0361

http://www.wi-ei.org/GBH/index.htm

jimes@wi-ei.org

\begin{tabular}{|ll|}
\hline Mandatory Requirements & \\
\hline Category & Points \\
\hline WI ENERGY STAR home & 10 Required \\
ENERGY StAR appliances & 1 Required \\
Erosion control plan (available in builder guidebook) & 1 Required \\
One recycled material (minimum 50\%) & 1 Required \\
No uncertified Lauan or other tropical hardwood plywood, \\
$\begin{array}{l}\text { doors, or trim } \\
\text { Homeowner handbook (prepared by program) }\end{array}$ & 1 Required \\
\hline
\end{tabular}




\section{Green Built Home (Wisconsin Environmental Initiative) continued}

\begin{tabular}{|ll|}
\hline Checklist Requirements & Maximum Possible Points \\
\hline Category & 13 \\
\hline Landscape conservation & 7 \\
\hline Energy use (general) & 5 \\
\hline Water conservation & 4 \\
\hline Materials selection & \\
\hline Energy use & 14 \\
Insulation and air sealing & 9 \\
Glazing & 17 \\
Mechanical systems & 15 \\
Indoor air quality & 14 \\
Water heating & 3 \\
Indoor water conservation & 7 \\
Appliances & 11 \\
Lighting & 6 \\
Integrated climatic design & \\
\hline Materials & 10 \\
Below grade & 18 \\
Structural frame & 16 \\
Envelope & 5 \\
Insulation & 3 \\
Roof & 5 \\
Subfloor & 15 \\
Finish floor & 14 \\
Doors, cabinets, and trim & 6 \\
\hline Finishes and adhesives & 15 \\
\hline Waste management & $\mathbf{2 3 2}$ \\
\hline Total Possible Points & \\
\hline
\end{tabular}




\section{Build A Better Kitsap Home Builder Program (Kitsap HBA, Washington)}

\section{Overview:}

Builders qualify at a one-, two-, or three-star level determined by mandatory criteria, plus points awarded from a checklist. Checklist items are generally weighted from 1 to 3 points.

\section{Certification method:}

Self-certification checklist (plus mandatory one-time program orientation)

Levels of certification:

One-, two-, or three-stars $(\star)$

Qualification:

$\star \quad$ All mandatory requirements, plus 10 points from Section 2 through 8 of checklist.

$\star$ All mandatory requirements, plus 30 points from Section 2 through 8 of checklist. Earn at least 3 points from each section.

$\star \star \star$ All mandatory requirements, plus 30 points total from Section 2

Year of inception: through 8 of checklist. Attend a workshop within 1 year of certification.

\section{Number of builders:} 27

Incentives offered to builders to participate:

Marketing such as billboards, Internet marketing, print brochures

Number of homes constructed to date:

$$
278
$$

Contact information:

Build a Better Kitsap

Art Castle

5251 Auto Center Way

Bremerton, WA 98312-3319

p. $360-479-5778$

f. 360-479-0313

acastle@kitsaphba.com

http://www.kitsaphba.com

\begin{tabular}{|ll|}
\hline Mandatory Requirements & \\
\hline Category & Points \\
\hline Meet Washington State energy code & Required \\
Meet Washington State ventilation/indoor air quality code & Required \\
Meet Washington State water use efficiency standards & Required \\
Program orientation (one time only) & Required \\
Provide "Operations and Maintenance Kit" & Required \\
Checklist Requirements & \\
Section & Maximum Possible Points \\
\hline Site protection & 18 \\
Site design & 22 \\
Reduce & 20 \\
Reuse & 8 \\
Recycle & 14 \\
Resource-efficient material selection & 31 \\
Maximize energy efficiency & 43 \\
Indoor air quality and health & 58 \\
Manage hazardous waste & 9 \\
Promote responsible operation and maintenance & 16 \\
\hline Total Possible Points & $\mathbf{2 3 9}$ \\
\hline
\end{tabular}




\section{EarthCraft House (Greater Atlanta HBA, Georgia)}

\section{Overview:}

Places emphasis and value on providing training and technical assistance to builders. Requires energy efficiency, plus other green features. All homes are inspected and blower-door tested by specially trained EarthCraft House inspectors. Checklist is weighted toward features that have the most environmental benefits. Allows bonus points for proximity to mass transit, PV or solar hot water, or other innovations. Includes a workbook for builders to educate about various items on the checklist.

\section{Certification method:}

Required third-party inspection, plus self-certification worksheet

Levels of certification:

One

Qualification:

Certification requires 150 points from the checklist out of 489 total possible points

Year of inception: 1999

Number of builders: 89

Incentives offered to builders to participate:

All first inspections are free to the builder, some incentives to homeowners in the form of reduced closing costs and lower interest rate mortgages, other loan assistance programs.

Number of homes constructed to date: 500

Contact information: EarthCraft House Greater Atlanta Home Builders Association, Inc. P.O. Box 450749 Atlanta, GA 31145 p. $770-938-9900$ f. $770-934-8363$ earthcraft@earthcrafthouse.com http://www.earthcrafthouse.com

"EarthCraft House renovation is perhaps the most exciting new aspect to our program. We've worked with six of the city's top renovators to outline how the program might work. We're currently in the pilot state, and so far the response just through word of mouth has been amazing. We plan to officially launch this part of the program in mid summer [2002] with a detailed workbook, and special training class."

Jim Hackler

EarthCraft House Greater Atlanta HBA 


\begin{tabular}{|c|c|}
\hline \multicolumn{2}{|l|}{ Checklist Requirements } \\
\hline Category & Maximum Possible Points \\
\hline Site planning & 45 \\
\hline Tree protection and planting measures & 15 \\
\hline Energy efficient building envelope and systems & 90 (for Energy Star certification) \\
\hline Energy measures & $\begin{array}{l}\text { HOuse must either be ENERGY } \\
\text { STAR certified or must get at } \\
\text { least } 75 \text { points from the follow- } \\
\text { ing "energy measures" below }\end{array}$ \\
\hline Air leakage test & 35 \\
\hline Air sealing measures & 30 \\
\hline Insulation & 50 \\
\hline Windows & 32 \\
\hline Heating and cooling equipment & 42 \\
\hline Ductwork/air handler & 58 \\
\hline Energy efficient lighting/appliances & 12 \\
\hline Resource efficient design & 26 \\
\hline \multicolumn{2}{|l|}{ Resource efficient building materials } \\
\hline Recycled/natural content materials & 10 \\
\hline Advanced products & 29 \\
\hline Durability & 15 \\
\hline \multicolumn{2}{|l|}{ Waste management } \\
\hline Waste management practices & 14 \\
\hline Recycle construction waste & 15 \\
\hline \multicolumn{2}{|l|}{ Indoor air quality } \\
\hline Combustion safety & 30 \\
\hline Moisture control & 13 \\
\hline Ventilation & 26 \\
\hline Materials & 13 \\
\hline Water-indoor use & 19 \\
\hline Water-outdoor use & 35 \\
\hline Homebuyer education/opportunities & 26 \\
\hline Builder operations & 13 \\
\hline Bonus points (mass transit, brownfield development, etc.) & $55+$ \\
\hline
\end{tabular}

\section{Total Possible Points}




\section{Built Green ${ }^{T M}$ Colorado (HBA of Metro Denver)}

\section{Overview:}

Comprehensive checklist that requires builders to meet the Energy Efficiency Minimum Requirement and then reach a cumulative point total of 70.

\section{Certification method:}

Self-certification checklist; $5 \%$ of all residential homes are inspected on random basis by third-party services.

Levels of certification:

One

Qualification:

Builders must have 70 points; the points can come from anywhere in the checklist.

Year of inception:

1995

Number of builders:

111

Incentives offered to builders to participate:

Market distinction, education, TV ads

Number of homes constructed to date:

9,646

\section{Contact information:}

Built Green Colorado

HBA of Metro Denver

1400 S. Emerson

Denver, CO 80210

p. $303-778-1400$

f. 303-733-9440

info@builtgreen.org

http://www.builtgreen.org 


\begin{tabular}{|ll|}
\hline Checklist Requirements & Maximum Possible Points \\
\hline Category & 3 \\
\hline Energy requirement (Required) & \\
\hline Energy efficiency & 60 \\
Envelope & 158 \\
Mechanical systems & 43 \\
Water heating & 34 \\
Appliances & 16 \\
Lighting & \\
\hline Materials & 33 \\
Foundation & 72 \\
Structural frame & 10 \\
Subfloor & 13 \\
Windows & 8 \\
Doors & 16 \\
Insulation & 34 \\
Exterior wall finishes & 14 \\
Roof & 31 \\
Finish floor & 14 \\
\hline Cabinetry and trim & \\
\hline Health and safety & 95 \\
\hline Indoor air quality & 17 \\
\hline Resource conservation & 9 \\
Land use & 16 \\
Materials reduction & 7 \\
Materials re-use & 35 \\
Waste reduction and recycling & 738 \\
Water & \\
\hline Total Possible Points & \\
\hline & \\
\hline
\end{tabular}




\section{BuiltGreen ${ }^{T M}$ (MBA of King and Snohomish Counties, Washington)}

Overview:

Comprehensive checklist with weighted items

Certification method:

Self-certification checklist

Levels of certification:

One-, two-, or three-stars $(\star)$

Qualification:

$\star \quad$ Attend program orientation; meet mandatory green codes and regulations; earn 25 points from checklist; prepare and post a jobsite recycling program; and provide an "Operations and Maintenance Kit."

$\star$ * One star requirements, plus 75 additional points (100 points minimum) from checklist with at least 6 points from each section; attend a Built Green workshop within 1 year of certification.

$\star \star \star$ Meet two-star requirements plus 105 additional points

Year of inception: (180 points minimum).

$$
2000
$$

Number of builders:

9 participating

Incentives offered to builders to participate:

Access to marketing tools

Number of homes constructed to date:

1,600

Contact information:

Built Green

Master Builders Association of King and Snohomish Counties

2155112 th Avenue, NE

Bellevue, WA 98004

p. $425-451-7920$

builtgreen@mba-ks.com

http://www.builtgreen.net

\begin{tabular}{|ll|}
\hline Mandatory Requirements & \\
\hline Category & Maximum Possible Points \\
\hline Meet Washington State water use efficiency standards & Required \\
\hline Meet stormwater/site development standards & Required \\
\hline Meet Washington State ventilation/indoor air quality code & Required \\
\hline Meet Washington State energy code & Required \\
\hline Provide Homeowner with Operations and Maintenance kit & Required \\
Checklist Requirements & \\
Site and water & \\
Overall & 13 \\
Protect sites natural features & 22 \\
Protect natural processes on-site & 58 \\
Eliminate water pollutants & 37 \\
Design alternatives & 25 \\
\hline
\end{tabular}




\begin{tabular}{|c|c|}
\hline \multicolumn{2}{|l|}{ Energy efficiency } \\
\hline Envelope & 128 \\
\hline Heating/cooling & 20 \\
\hline Water heating & 5 \\
\hline Lighting & 6 \\
\hline Efficient design & 3 \\
\hline Alternative systems & 15 \\
\hline \multicolumn{2}{|l|}{ Health and indoor air quality } \\
\hline Overall & 25 \\
\hline Jobsite operations & 19 \\
\hline Layout and material selection & 50 \\
\hline Moisture control & 9 \\
\hline Air distribution and filtration & 21 \\
\hline HVAC equipment & 30 \\
\hline \multicolumn{2}{|l|}{ Materials efficiency } \\
\hline Overall & 40 \\
\hline \multicolumn{2}{|l|}{ Jobsite operations } \\
\hline Reduce & 9 \\
\hline Reuse & 13 \\
\hline Recycle & 40 \\
\hline \multicolumn{2}{|l|}{ Design and material selection } \\
\hline Overall & 10 \\
\hline Framing & 29 \\
\hline Foundation & 4 \\
\hline Subfloor & 1 \\
\hline Doors & 5 \\
\hline Finish floor & 15 \\
\hline Interior walls & 2 \\
\hline Exterior walls & 8 \\
\hline Windows & 2 \\
\hline Cabinetry and trim & 12 \\
\hline Roof & 7 \\
\hline Insulation & 5 \\
\hline Other exterior & 10 \\
\hline \multicolumn{2}{|c|}{ Promote environmentally friendly homeowner Operation and Mainenance } \\
\hline Water conservation-outdoor & 40 \\
\hline Water conservation-indoor & 31 \\
\hline Eliminate water pollutants & 5 \\
\hline \multicolumn{2}{|l|}{ Energy } \\
\hline Heating/cooling & 9 \\
\hline Water heating & 19 \\
\hline Appliances & 10 \\
\hline Efficient lighting & 9 \\
\hline Health and indoor air quality & 3 \\
\hline Recycling & 6 \\
\hline Total Possible Points & 830 \\
\hline
\end{tabular}




\section{Green Home Designation (Florida Green Building Coalition)}

\section{Overview:}

Program that features a comprehensive, weighted checklist of efficiency measures. Gives value to meeting Florida energy code, plus additional energy points for HERS rating above 80 and design, appliances, lights, and amenities. Also has categories for water, site, health, materials, and disaster mitigation.

"The most

valuable aspect

of Florida's

Green Building

Program is the

one-on-one

assistance

available to

builders through

program

Certifying

Agents, and the

Building America

Industrialized

Housing

Partnership."

\section{Eric Martin}

Florida Green

Building

Coalition

\section{Certification method:}

Mix of self-certified and inspector-certified items. Some items require special submittals for verification

Levels of certification:

One level

Qualification:

Builders must achieve a minimum number of points in each category to encourage diversity and to consider the house as a system. However, if there is a deficit in one category, it can be amended if the deficiency is added to the total minimum score of 200. For example, if the home achieves only 10 points in a category with a minimum of 15 required, the builder can still qualify for a Green Building Designation if the total number of points is, at minimum, 205.

Year of inception: 2001

Number of builders: 15

Incentives offered to builders to participate: Discount on per-home registration fee

Number of homes constructed to date:

$$
2
$$

Contact information:

Green Home Designation

Eric Martin, Research Engineer

Florida Solar Energy Center

1679 Clearlake Road

Cocoa, FL 32922

p. $321-638-1450$

f. $321-638-1439$

info@floridagreenbuilding.org

http://www.floridagreenbuilding.org

\begin{tabular}{|c|c|}
\hline \multicolumn{2}{|l|}{ Mandatory Requirements } \\
\hline Category & Maximum Possible Points \\
\hline \multicolumn{2}{|l|}{ Prerequisite 1 (use at least one measure) } \\
\hline $\begin{array}{l}\text { Sanitation system that reduces/eliminates chlorine use } \\
\quad \text { (salt water, ionization, etc.) } \\
\text { Pool cover } \\
\text { Solar pool heating system } \\
\text { Efficient pool pumping } \\
\text { No swimming pool or spa }\end{array}$ & $\begin{array}{l}\text { Required } \\
\text { Required } \\
\text { Required } \\
\text { Required } \\
\text { Required }\end{array}$ \\
\hline \multicolumn{2}{|l|}{ Prerequisite 2 (use at least one measure) } \\
\hline $\begin{array}{l}\text { Use of native aquatic vegetation in shoreline area } \\
\text { Low-maintenance plants placed between lawn } \\
\text { and shoreline; no turf adjacent to water } \\
\text { Use of terraces, swales, or berms to slow stormwater } \\
\text { movement into water body } \\
\text { Home site does not border natural water body }\end{array}$ & $\begin{array}{l}\text { Required } \\
\text { Required } \\
\text { Required } \\
\text { Required }\end{array}$ \\
\hline
\end{tabular}




\section{Checklist Requirements}

Energy (building envelope/systems)

Codes/Ratings (both inspector-certified) Design

Energy (appliances, lights, amenities)

Energy-efficient appliances/amenities

Energy-efficient lighting

(100 min/150 max)

150

19

(10 min/25 max)

9

19

\begin{tabular}{|c|c|}
\hline Water & (15 min/40 max) \\
\hline Appliances & 12 \\
\hline Greywater reuse & 4 \\
\hline Rainwater harvesting & 4 \\
\hline Installed landscape & 25 \\
\hline Installed irrigation & 14 \\
\hline Site & $(10 \mathrm{~min} / 30 \mathrm{max})$ \\
\hline Native tree and plant preservation & 15 \\
\hline On-site use of cleared materials & 2 \\
\hline Erosion control/topsoil preservation & 5 \\
\hline Drainage/retention & 10 \\
\hline Health & $(10 \mathrm{~min} / 30 \mathrm{max})$ \\
\hline Combustion & 10 \\
\hline Moisture control & 7 \\
\hline Ventilation & 20 \\
\hline Source control (materials) & 9 \\
\hline Cleanability & 4 \\
\hline Universal design & 4 \\
\hline Materials & $(10 \mathrm{~min} / 45 \mathrm{max})$ \\
\hline Structure & 14 \\
\hline Sub-assembly & 7 \\
\hline Partitions / trim & 4 \\
\hline Finishes & 4 \\
\hline Durability & 6 \\
\hline Waste management & 12 \\
\hline Disaster mitigation & (5 min/30 max) \\
\hline Hurricane (wind, rain, storm surge) & 20 \\
\hline Flood (check all 4 to receive 5 points) & 5 \\
\hline Wild fire (check all 3 to receive 5 points) & 5 \\
\hline Termites (check all 12 to receive 10 points) & 10 \\
\hline General & (0 min/50 max) \\
\hline Small house credit & 50 \\
\hline Renewable power generation & 20 \\
\hline Reconfigurability & 6 \\
\hline Lot choice & 10 \\
\hline Other & 14 \\
\hline Total Possible Points & 400 \\
\hline
\end{tabular}




\section{City of Boulder Green Points (Colorado)}

\section{Overview:}

Checklist of features that are weighed.

Certification method:

City- or self-certification checklist (method required is specified for each checklist item).

Levels of certification: One

Qualification:

Point requirements are based on square footage. Homes smaller than or equal to 1,500 square feet need 50 points. Homes between 1,501 and 2,500 square feet need 65 points. Homes larger than 2,501 square feet need one additional point for each 50 additional square feet up to the maximum allowable points. The program also covers remodeling and additions over 500 square feet.

Year of inception: 1997

Number of builders:

38 have gone through training. All builders in Boulder participate because it is mandatory.

Incentives offered to builders to participate: Must participate to get building permit.

Number of homes constructed to date: Approximately 116

\section{Contact information:}

Mike Weil

Director, Energy Programs Coordinator

City of Boulder

Office of Environmental Affairs

P.O. Box 791

Boulder, CO 80306

p. 303-441-4191

f. 303-441-4070

weilm@ci.boulder.co.us

http://www.ci.boulder.co.us/environmentalaffairs/green_points/

\section{Checklist Requirements}

\begin{tabular}{|ll|}
\hline Category & Maximum Possible Points \\
\hline Construction/Demolition and Use of recycled materials & 29 \\
\hline Land use and Water conservation & 25 \\
\hline Framing & 30 \\
\hline Energy code measures & 113 \\
\hline Plumbing & 5 \\
\hline Electrical & 10 \\
\hline Insulation & 34 \\
\hline HVAC & 51 \\
\hline Solar & 79 \\
\hline Indoor air quality & 48 \\
\hline Innovation & 10 \\
\hline Total Possible Points & Approximately $\mathbf{4 3 4}$ \\
\hline
\end{tabular}




\section{Green Building Program, Austin Energy (Texas)}

\section{Overview:}

Comprehensive, weighted checklist

Certification method:

Self-certification

\section{Levels of certification:}

One-, two-, three-, four-, and five-stars ( $\star$ )

Qualification:

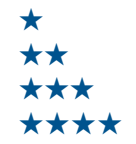

40-59 points

60-89 points

90-129 points

$\star \star \star \star$ 130-179 points (must include blower-door test, duct-blaster test, or ducts located in conditioned space and combustion/ backdraft test)

$\star \star \star \star \star 180$ or more points (including requirements listed in four stars)

Year of inception:

1990

Number of builders:

111

Incentives offered to builders to participate:

Training (e.g., monthly seminars), support services, plan reviews, one-on-one consultation, marketing

Number of homes constructed to date:

2,475

Contact information:

Richard Morgan

P.O. Box 1088

Austin, TX 78767

p. 512-505-3709

f. 512-505-3711

Richard.morgan@austinenergy.com

http://www.ci.austin.tx.us/greenbuilder

\begin{tabular}{|ll|}
\hline Mandatory Requirements & \\
\hline Category & Maximum Possible Points \\
\hline Durable finish (min. 50-year warranty) & Required \\
\hline One recycled content material (min 50\%) & Required \\
\hline Meet City of Austin Building and Energy Code & Required \\
\hline $\begin{array}{ll}\text { Efficient and effective cooling and dehumidification } \\
\text { system }\end{array}$ & Required \\
\hline Two ceiling fans & Required \\
\hline Meet City of Austin Building Code requirements & Required \\
\hline No vapor barrier on inside perimeter wall & Required \\
\hline One-inch minimum pleated filter on HVAC system & Required \\
\hline Low-VOC paints on interior & Required \\
\hline If termite control used, pyrethroid or borate based & Required \\
\hline Any planting beds mulched to min 2" depth & Required \\
\hline Rating certificate and homeowner information packet & \\
\hline given to homeowner & Required \\
\hline Rating submitted for all homes in greater Austin Area & Required \\
\hline
\end{tabular}




\section{Green Building Program, Austin Energy continued}

\begin{tabular}{|ll|}
\hline \multicolumn{1}{|l|}{$\mid$} \\
\hline Checklist Requirements & Maximum Possible Points \\
\hline Section & \\
\hline Energy & 43 \\
Design & 22 \\
Thermal envelope & 38 \\
Heating, cooling, water heating & 13 \\
\hline Lighting and appliances & \\
\hline Materials & 15 \\
Design, structure & 16 \\
Finish materials & 10 \\
\hline Excess jobsite resources & \\
\hline Water & 6 \\
Indoor & 28 \\
\hline Outdoor & 21 \\
\hline Health, Safety & 18 \\
Molds, mites, fibers & 7 \\
Chemical outgassing & 2 \\
Combustion gases & 7 \\
\hline Electromagnetic fields & 28 \\
\hline Integrated pest management & $\mathbf{2 7 4}$ \\
\hline Community & \\
\hline Total Possible Points & \\
\hline & \\
\hline
\end{tabular}

"After 10 years of promoting Green Building in Austin we now have home buyers asking for 'green' features in the homes they buy. We have achieved this through a long-term effort to educate the consumer about the benefits of green building....Builders [also benefit] from the technical assistance they get from us. This assistance ranges from free monthly seminars for members of our program to individual sessions with our staff to help them achieve a higher level of energy efficiency, comfort, and durability in their homes."

\section{Richard Morgan}

Austin Energy Green Building Program 


\section{City of Scottsdale Green Building Program (Arizona)}

\section{Overview:}

Weighted rating (checklist) that emphasizes a system's approach by requiring 26 prerequisites. After meeting these requirements, projects get points from various rating categories. Projects qualify as "entry level" (26 points from the rating categories) or "advanced level" (56 points from the rating categories).

\section{Certification method:}

Checklist items verified by inspector. Certification and Homeowners Guide provided at completion of project.

\section{Levels of certification:}

Entry and advanced

Qualification:

63 points out of 368

Year of inception: 1998

Number of builders:

47

Incentives offered to builders to participate:

Expedited plan review: permitting time reduced by half

Number of homes constructed to date: 129

Contact information:

Anthony Floyd

7506 East Indian School Road

Suite 125

Scottsdale, AZ 85251

p. $480-312-4202$

f. $480-312-7314$

afloyd@ci.scottsdale.az.us

http://www.ci.scottsdale.az.us/greenbuilding/

\begin{tabular}{|lll|}
\hline $\begin{array}{l}\text { Requirements } \\
\text { Category }\end{array}$ & Required Elements & $\begin{array}{l}\text { Maximum Possible } \\
\text { Points }\end{array}$ \\
\hline Site use & 1 & 26 \\
\hline Structural elements & 1 & 23 \\
\hline Building envelope & 5 & 63 \\
\hline Heating, cooling, and ventilation & 6 & 59 \\
\hline Indoor air quality & 4 & 21 \\
\hline Electrical power, lighting, and appliances & 3 & 25 \\
\hline Plumbing system & 3 & 38 \\
\hline Roofing & - & 12 \\
\hline Exterior finishes & - & 12 \\
\hline Interior finishes & 1 & 10 \\
\hline Interior doors, cabinetry, trim & - & 15 \\
\hline Finish floor & 1 & 13 \\
\hline Pools and spas & - & 26 \\
\hline Solid waste & 1 & 7 \\
\hline Special options & - & 18 \\
\hline Total Possible Points & $\mathbf{2 6}$ & $\mathbf{3 6 8}$ \\
\hline
\end{tabular}


New Mexico Building America Partner Program

(HBA of Central New Mexico)

\section{Overview:}

Minimum standards for energy conservation (prescriptive or performance-

based compliance), indoor air quality, water conservation, building materials conservation, solid waste reduction through recycling, and testing. No checklist or optional items. In addition to addressing Building America objectives of performance-based goals for indoor air quality, energy conservation, and water conservation, this program addresses solid waste reduction and material conservation.

Certification method:

Levels:

Third-party testing done by HERS raters

$$
\text { One }
$$

Qualification:

Two technical seminars and two public seminars

Year of Inception:

$$
2001
$$

Number of builders:

15

Incentives offered to builders to participate: Marketing niche

Number of homes constructed to date: 830

Contact information:

HBA of Central New Mexico

Building America Partner Program

Lindsay Chism

PO Box 1881

Los Lunas, NM 87031

p. $505-866-6479$

f. 505-565-8207

Idcconsulting@aol.com

http://www.hbacnm.com/green_builder 


\section{County of Santa Barbara Innovative Building Review Program (California)}

\section{Overview:}

Free program that advises developers on how to make developments more energy efficient. Includes a few green features beyond energy efficiency. Incentives to meeting targets include expedited plan review, 50\% reduction in energy plan-check fee, marketing materials, and eligibility for Energy-Efficient Building of the Year.

\section{Certification method:}

Plan review (by committee at regularly scheduled meetings) and self-certification checklist.

Levels of certification:

Target 1, 2, or 3

Qualification:

Target 1: $20 \%$ better than Title 24 (California Energy Code);

5 points from checklist.

Target 2: $\quad 30 \%$ better than Title $24 ; 12$ points from checklist.

Target 3: $\quad 40 \%$ better than Title 24; 30 points from checklist.

Year of inception:

1995

Number of builders:

60

Incentives offered to builders to participate:

Expedited review and reduced fees for checking energy plan.

Number of homes constructed to date: 890

\section{Contact information:}

County of Santa Barbara

Innovative Building Review Program

Kathy Pfeifer

30 E. Figueroa Street, 2nd Floor

Santa Barbara, CA 93101-2709

p. $805-568-2507$

f. 805-568-2522

kathypm@co.santa-barbara.ca.us

http://www.silcom.com/ sbcplan/ibdrc.html

\begin{tabular}{|ll|}
\hline Mandatory Requirements & \\
\hline Category & Maximum Possible Points \\
\hline Exceed Title 24 requirements by 20\% & Required (Target 1) \\
Exceed Title 24 requirements by 30\% & Required (Target 2) \\
Exceed Title 24 requirements by 40\% & Required (Target 3) \\
Checklist Requirements & \\
\hline Category & Maximum Possible Points \\
\hline Energy & 51 \\
Siting & 4 \\
Summer shading and wind protection & 52 \\
Non-energy related building techniques & 23 \\
\hline Total Possible Points & $\mathbf{1 3 0}$ \\
\hline
\end{tabular}




\section{Build a Better Clark (Clark County, Washington HBA)}

\section{Overview:}

Builders qualify at a one-, two-, or three-star level determined by mandatory criteria, plus points awarded from a checklist. Checklist items are generally weighted from 1 to 3 points.

\section{Certification method:}

Self-certification checklist (plus mandatory one-time program orientation)

\section{Levels of certification:}

One-, two-, or three-stars $(\star)$

\section{Qualification:}

$\star \quad$ All mandatory requirements.

$\star \star \quad$ All of one-star requirements, plus earn additional 50 points total. Attend a workshop within past 12 months.

$\star \star \star \quad$ Two-star requirements, plus an additional 40 points total (for a minimum total of 90 points). Attend a workshop

Year of inception: within past 12 months.

$$
1999
$$

\section{Number of builders:}

16

Incentives offered to builders to participate:

In development

Number of homes constructed to date:

$$
26
$$

Contact information:

Build a Better Clark

Attn: Mary Gould

5007 NE St. John's Road

Vancouver, WA 98661

p. $360-694-0933$

f. 360-694-1606

joel@cchba.com

http://www.cchba.com/build_a_better_clark_page.htm 


\begin{tabular}{|ll|}
\hline Mandatory Requirements & \\
\hline Category & Maximum Possible Points \\
\hline Meet Washington State energy code & Required \\
\hline Meet Washington State ventilation/indoor air quality code & Required \\
\hline Meet Washington State water use efficiency standards & Required \\
\hline Prepare a job-site recycling plan and post on-site & Required \\
\hline Use at least one recycled-content building product & Required \\
\hline Provide a "Homeowner's Kit" & Required \\
\hline Program orientation (one time only) & Required \\
\hline Checklist Requirements & \\
\hline Category & Maximum Possible Points \\
\hline Treat site appropriately & \\
\hline Site protection & 19 \\
Site design & 20 \\
\hline Prevent waste & \\
Reduce & 23 \\
Reuse & 8 \\
\hline Recycle & 12 \\
\hline Resource-efficient material selection & 32 \\
\hline Maximize energy efficiency & 46 \\
\hline Indoor air quality and health & 56 \\
\hline Manage hazardous waste & 9 \\
\hline Promote responsible operation and maintenance & 16 \\
\hline Total Possible Points & $\mathbf{2 4 1}$ \\
\hline & \\
\hline
\end{tabular}




\section{Earth Advantage ${ }^{T M}$ Program (Portland General Electric, Oregon)}

\section{Overview:}

Earth Advantage is a utility-run program that provides marketing and technical support to builders. The program starts with a plan review by an Earth Advantage technical specialist. The specialist conducts on-site inspections to check for proper installation of materials. Two diagnostic tests are performed: one in the early stages of building to test the duct air loss and the second-a blower door test-is conducted when the home is complete. A certificate is given that lists the features for the home, the appliance ratings, and the diagnostic test results.

\section{Certification method:}

Points worksheet and onsite inspections

\section{Levels of certification:}

One

Qualification:

For the house to be certified, the builder must achieve a minimum of 50 points in each of the four following categories:

1) Energy Efficiency

2) Healthier Indoor Air

3) Environmental Responsibility

4) Resource Efficiency

House must also pass two performance tests (duct-blast and blower-door). To ensure that the house meets the requirement for performing $15 \%$ better than the Oregon energy code, core measures are required in each of the five following categories that include energy-efficiency points:

1) Shell Construction

2) HVAC/Duct Sealing

3) Water Heating

4) Lighting

5) Appliances.

Year of inception: 1999

Number of builders:

$$
33
$$

Incentives offered to builders to participate:

Marketing, advertising, others to be developed

Number of homes constructed to date: $100+$

\section{Contact information:}

Duane Woik

16280 SW Upper Boones Ferry Road

Portland, OR 97224

p. $503-603-1733$

f. $503-603-1710$

http://www.earthadvantage.com 


\begin{tabular}{|c|c|c|c|c|}
\hline \multirow[t]{2}{*}{ Checklist Requirements } & \multicolumn{4}{|c|}{ Maximum Possible Points } \\
\hline & \multicolumn{4}{|c|}{ Energy and Environmental Categories } \\
\hline $\begin{array}{l}\text { Construction } \\
\text { Categories }\end{array}$ & $\begin{array}{l}\text { Energy } \\
\text { Efficiency }\end{array}$ & $\begin{array}{l}\text { Healthier } \\
\text { Indoor Air }\end{array}$ & $\begin{array}{l}\text { Environmental } \\
\text { Responsibility }\end{array}$ & $\begin{array}{l}\text { Resource } \\
\text { Efficiency }\end{array}$ \\
\hline Shell construction & 134 & 56 & 72 & 118 \\
\hline HVAC & 160 & 160 & 20 & 64 \\
\hline Water heating & 69 & 0 & 30 & 32 \\
\hline Lighting & 6 & 0 & 12 & 8 \\
\hline Appliances & 12 & 0 & 8 & 6 \\
\hline Foundation & 0 & 34 & 12 & 26 \\
\hline Siding & 0 & 58 & 44 & 68 \\
\hline Roofing & 0 & 22 & 18 & 36 \\
\hline Insulation material & 0 & 0 & 4 & 10 \\
\hline Interior surfaces & 0 & 4 & 4 & 10 \\
\hline Surface coating & 0 & 54 & 24 & 28 \\
\hline Cabinets & 0 & 42 & 28 & 28 \\
\hline Countertops & 0 & 32 & 12 & 16 \\
\hline Casework & 0 & 18 & 12 & 8 \\
\hline Stove/fireplace & 0 & 22 & 4 & 0 \\
\hline Flooring & 0 & 46 & 36 & 42 \\
\hline Finish plumbing & 0 & 0 & 8 & 6 \\
\hline Land and water & 0 & 0 & 144 & 2 \\
\hline Waste management & 0 & 0 & 28 & 16 \\
\hline Total Possible Points & 381 & 548 & 520 & 524 \\
\hline
\end{tabular}




\section{G/Rated (City of Portland, Oregon)}

\section{Overview:}

Builders participating in this city-operated program are awarded points from a checklist for certification. Projects are jury-reviewed and are selected for publicity purposes.

\section{Certification method:}

Inspection, plus self-certification weighted checklist.

Levels of certification:

One

Qualification:

Case Study

Year of inception:

2001

Number of builders:

Not tracked

Incentives offered to builders to participate:

$\$ 3,000$ grants for qualified projects that serve as case studies to be shared with the community.

Number of homes constructed to date:

35 (case studies)

Contact information:

Mike O'Brien

City of Portland Office of Sustainable Development

Green Building Specialist

p. 503-823-5494

mobrien@ci.portland.or.us

http://www.green-rated.org 


\section{Checklist Requirements}

\section{Category \\ Maximum Possible Points}

Sustainable sites

Alternative transportation

Erosion and sediment control 2

Stormwater management 3

Healthy and water-efficient landscaping $\quad 14$

House design $\quad 10$

Energy efficiency

Building envelope

Heating and cooling 13

Water heating 5

Appliances and lights $\quad 5$

Renewable energy 18

Materials and resources

Building materials 11

Efficient structural systems 20

Waste reduction and management 11

$\begin{array}{ll}\text { Reducing pollutant sources } & 13\end{array}$

Ventilation 9

Air cleaning 5

Reduce toxins in yard 4

Innovations 23

Partnerships 9

New technologies 5

Total Possible Points

193 
Home Builders Association of Greater Kansas City (Missouri)

\section{Overview:}

Self-certification worksheets. The individual items are not weighted.

Certification method:

Builders enroll in the Home Builders Association of Greater Kansas City Build Green Council and take an orientation class. Then, the builder can enroll individual homes in one of four possible levels (Platinum, Gold, Silver, or Bronze). Eight hours of classroom instruction per year are required. Each home above the bronze level requires a home energy rating. Only the builder and homebuyer receive and use the guideline, nothing is sent into the HBA.

Levels of certification:

Platinum, Gold, Silver, or Bronze

Qualification:

There are five categories of guidelines: Site, Energy, Materials, Indoor Air Quality, and Recycling. The energy aspect of the guidelines are performancebased, rather than prescriptive (i.e., the guidelines require an energy rating, but the guidelines do not dictate how the builder reaches the appropriate energy rating level). The guidelines offer suggestions that the builder can check off (e.g., sealed combustion, direct vent water heater) and submit to the HBA and also give to the home buyer. The Bronze level does not require a home energy rating. An energy rater does not have to be contacted-the builder can self-certify that the home meets the 1993 MEC.

Year of inception: 2002

Number of builders: TBA

Incentives offered to builders to participate:

Home tours, use of logo on house listing, other marketing

Number of homes constructed to date: TBA

Contact information:

Stan Parsons

Staff Coordinator

HBA of Greater Kansas City

600 East 103rd Street

Kansas City, MO 64131

p. $816-942-8800 \times 231$

f. $816-942-8367$

stan@kchba.org

http://www.kchba.org 


\section{City of Frisco (Texas) Green Building Program}

\section{Overview:}

This is one of the only programs in the country where all new residential homes (platted after May 23, 2001) must meet or exceed the green building program's criteria (i.e., it is not a voluntary program). The individual items are not weighted in the minimum standards list.

\section{Certification method:}

Performance-based program

Levels of certification:

One

\section{Qualification:}

There are four categories of guidelines: Energy Efficiency, Water Conservation, Indoor Air Quality, and Waste Recycling. The Energy Efficiency aspect of the standards are performance-based, rather than prescriptive. The standards require that the house meets or exceeds the EnERGY Star Homes designation, but they do not dictate how the builder reaches the appropriate energy rating level. There are minimum standards for the other three categories.

Year of inception: 2001

Number of builders: 40

Incentives offered to builders to participate: Mandatory participation

Number of homes constructed to date: 1,600 units are in the queve

\section{Contact information:} Jeff Witt

City of Frisco

6875 Main Street

Frisco, TX 75034

p. $972-335-5540 \times 145$

f. $972-335-5549$

jwitt@ci.frisco.tx.us

http://www.ci.frisco.tx.us/planning/greenbuilding_index.htm 
Hawaii BuiltGreen ${ }^{\mathrm{TM}}$

Overview:

Comprehensive checklist with weighted items

Certification method:

Self-certification checklist

Levels of certification:

One-, two-, and three-stars $(\star)$

Qualification:

* Attend a one-time program orientation; meet mandatory green codes and regulations; earn at least 35 points for naturally ventilated homes; earn at least 45 points for air-conditioned homes.

$\star \star \quad$ One-star requirements, plus 85 additional points (120 or 130 points total, respectively) from Sections 1 through 5 with at least 5 points from each section.

$\star \star \star$ Meet two-star requirements, plus 95 additional points (215 or 225 points total, respectively); attend green-building-related workshop or conference within past 12 months.

\section{Year of inception:}

TBA

Number of builders:

TBA

Incentives offered to builders to participate:

Identified in Parade of Homes directory, serve in speakers' bureau

Number of homes constructed to date:

TBA

Contact information:

Karen Nakamura

Executive Vice President

Building Industry Association of Hawaii

1727 Dillingham Blvd.

Honolulu, HI 96819

p. $808-847-4666 \times 203$

f. 808-842-0129

ktn@bia-hawaii.com

http://www.bia-hawaii.com/builtgreen/

\begin{tabular}{|ll|}
\hline Mandatory Requirements & \\
\hline Category & Maximum Possible Points \\
\hline No soil exposed during job (protected with mulch) & Required \\
\hline No fill in sensitive areas & Required \\
\hline Sensitive areas flagged and protected during construction Required \\
\hline Post-cleanup procedures for spills & Required \\
\hline Hazardous wastes separated and properly disposed of & Required \\
\hline Sediment traps installed for construction & Required \\
\hline No adverse impacts on adjoining properties or critical & \\
\hline areas during construction & Required \\
\hline Water quality monitoring during construction & Required \\
\hline Concrete trucks and pumps washed in designated areas & Required \\
\hline Low flow shower heads and sinks (2.5 gpm) & Required \\
\hline Low flow bath faucets (2.0 gpm) & Required \\
\hline Clothes dryer vented to outdoors & Required \\
\hline All wood used has approved chemical treatment & \\
\hline for termites & Required \\
\hline All cuts and drill holes in CCA-treated wood field-treated & Required \\
\hline Homeowner's operations and maintenance manual & Required \\
\hline Homeowner's outdoor landscaping manual & Required \\
\hline
\end{tabular}




\begin{tabular}{|c|c|}
\hline \multicolumn{2}{|l|}{ For Air-Conditioned Homes Only } \\
\hline House meets Hawaii MEC standards for A/C buildings & Required \\
\hline A/C system sized for efficient operation (not oversized) & Required \\
\hline Programmable thermostats provided & Required \\
\hline \multirow{2}{*}{\multicolumn{2}{|c|}{$\begin{array}{l}\text { Heat-trap installed or 1" pipe insulation on at least } \\
\text { first } 8 \text { feet of outlet pipe from water heater } \\
\text { Solar heater or heat pump for swimming pool heaters }\end{array}$}} \\
\hline & \\
\hline \multicolumn{2}{|l|}{ Checklist Requirements } \\
\hline \multicolumn{2}{|l|}{ Protecting Site } \\
\hline Design choices & 35 \\
\hline Job site operations & 15 \\
\hline Outdoor water conservation & 9 \\
\hline Bonus points & 10 \\
\hline \multicolumn{2}{|l|}{ Energy performance and comfort } \\
\hline Site & 15 \\
\hline Shell & 24 \\
\hline Openings & 43 \\
\hline Interior layout and finishes & 10 \\
\hline Mechanical venting and cooling & 21 \\
\hline A/C homes only & 23 \\
\hline Water Heating - distribution & 25 \\
\hline \multicolumn{2}{|l|}{ Indoor water conservation (double points if } \\
\hline rainwater collection is not required) & 12 \\
\hline Electric lighting & 16 \\
\hline Appliances & 17 \\
\hline Bonus points for custom homes & 10 \\
\hline \multicolumn{2}{|l|}{ Health and indoor air quality } \\
\hline Floors & 38 \\
\hline Cabinetry and trim & 8 \\
\hline Interior walls & 8 \\
\hline Mechanical and other controls & 14 \\
\hline A/C Homes only & 11 \\
\hline Job site operations & 10 \\
\hline \multicolumn{2}{|l|}{ Durability and Materials Conservation } \\
\hline Design choices & 8 \\
\hline Termite details & 19 \\
\hline Framing & 24 \\
\hline Foundation & 9 \\
\hline Sub-floor & 2 \\
\hline Windows and doors & 10 \\
\hline Insulation & 4 \\
\hline Interior walls & 1 \\
\hline Finish floor & 19 \\
\hline Cabinetry and trim & 17 \\
\hline Roof & 5 \\
\hline Exterior finish & 8 \\
\hline Outdoor features & 12 \\
\hline Job site operations (triple points for custom homes) & 21 \\
\hline Bonus points & 25 \\
\hline Environmentally friendly homeowner O\&M & 11 \\
\hline Total Possible Points & 569 \\
\hline
\end{tabular}




\section{California Green Builder Program}

\section{Overview:}

The individual items are not weighted in the minimum standards list. However, for each of the four categories of guidelines, the program has established performance-based requirements.

\section{Certification method:}

Performance-based program

Levels of certification:

One

Qualification:

There are four categories of guidelines: Energy Efficiency, Air Quality, Waste Recycling, and Water Conservation. Below are the minimum requirements under the program:

Energy Efficiency: Meet EPA/DOE Energy STAR Homes efficiency levels; exceed a 15\% improvement over California Title 24 Energy Code.

Air Quality: Reduce air emissions by building to EnERGY STAR Homes efficiency level.

Waste Recycling: 50\% diversion from land fill job site waste. Where recycling and diversion are not available, builder agrees to adopt the Building Industry Institute Waste Recycling Guidelines and work with local jurisdictions to overcome local market barriers.

Water Conservation: $25 \%$ decrease in water use compared to typical

\section{Year of inception:} 1980s home.

$$
2001
$$

Number of builders:

$$
\text { TBA }
$$

Incentives offered to builders to participate:

Recognition, expedited plancheck, reduced fees, and expedited field check.

Number of homes constructed to date: TBA

Contact information:

Robert Raymer, P.E.

Bll Technical Director

1215 K Street, Suite 1200

Sacramento, CA 95814

p. 916-443-7933

http:www.thebii.org/cgbp.asp 


\section{Green Built Program (HBA of Greater Grand Rapids, Michigan)}

\section{Overview:}

Energy efficiency covered by participation in ENERGY STAR or American Lung Association Health (ALA) House. Checklist for additional points focuses on areas not addressed by ENERGY STAR or ALA programs.

\section{Certification method:} Independent auditor completes application

Levels of certification: One

Qualification:

Meet Energy StAR minimum requirements, plus 40 additional points from checklist

Year of inception: Spring 2002

Number of builders:

8, plus 6 associate members

Incentives offered to builders to participate: Use of Green Built logos; inclusion in list of builders distributed to general public inquiring about Green Building; names included in HBA marketing materials

Number of homes constructed to date: 4

\section{Contact information:}

Ann Dykema

Home \& Building Association of Greater Grand Rapids

2021 44th Street SE

Grand Rapids, MI 49508-5009

p. 616-281-2021

f. 616-281-4500

adykema@hbaggr.com

http://www.hbaggr.com

\section{Mandatory Requirements}

Category Maximum Possible Points

Attendance at training program

Four-Star ENERGY STAR rating

Required

Checklist Requirements

\section{Category}

Maximum Possible Points

Five-Star Energy Star Rating

1 for every ENERGY STAR point over

American Lung Association Health House ${ }^{\circledR}$ 80 , up to a maximum of 100

Land use 100 points

Water efficiency

13

Roofing

Framing/Decking

Foundation

Appliances

Lighting 


\section{Vermont Built Green (in progress)}

\section{Overview:}

Weighted checklist with minimum requirements. Points are accumulated for a total score.

Certification method:

Combination of self-certification and spot inspections.

Levels of certification:

Either "Vermont Built Green Certified" or "Vermont Built Green Certified with XX points"

Qualification:

TBA

Year of inception:

TBA

Number of builders:

N/A

Incentives offered to builders to participate:

ENERGY StAR certification

Number of homes constructed to date:

N/A

Contact information:

Richard Faesy

Vermont Energy Investment Corporation

255 S. Champlain Street

Burlington, VT 05401

p. 802-658-6060

f. 802-658-1643

rfaesy@veic.org

http://www.sover.net/ michaelh/bsr

\begin{tabular}{|ll|}
\hline Mandatory Requirements (draft) & \\
\hline Category & Maximum Possible Points \\
\hline $\begin{array}{l}\text { Siting and land use } \\
\text { Location }\end{array}$ & Required \\
Minimize damage to environment & Required \\
Promote community and security & Required \\
\hline Building design-efficient design & Required \\
\hline Quality/Durability-choose quality materials & Required \\
\hline Energy use & Required \\
Envelope and systems & Required \\
Lighting and appliances & Required \\
\hline Sustainable equipment & Required \\
\hline Resource impacts & Required \\
Resource efficient materials & Required \\
Reduce, reuse, recycle & Required \\
\hline $\begin{array}{l}\text { Encourage waste reuse and recycling for homeowners } \\
\text { Water efficiency }\end{array}$ & Required \\
\hline Occupant health/Indoor air quality & Required \\
\hline $\begin{array}{l}\text { Minimize sources of pollutants } \\
\text { Provide ventilation to remove generated pollutants }\end{array}$ & Required \\
\hline Occupant education and Operations and Maintenance & Requed \\
\hline
\end{tabular}




\section{Checklist Requirements}

Category Maximum Possible Points

Siting and land use

Location 18

Minimize damage to environment 31

Promote community and security $\quad 23$

Nature connection 3

Building design

Efficient design

7

Minimize house size

7

(multiplication factor for total checklist points. $2,001 \mathrm{ft}^{2}-2,500$

$\mathrm{ft}^{2}$ is $1.00 ; 2,000 \mathrm{ft}^{2}$ is 1.05 ; add

0.05 for each $100 \mathrm{ft}^{2}$ less than

$2,000 \mathrm{ft}^{2}$; subtract 0.05 for each $500 \mathrm{ft}^{2}$ over $2,500 \mathrm{ft}^{2}$ )

Quality/Durability-choose quality materials 39

Energy use

Envelope and systems 31

Lighting and appliances $\quad 18$

Sustainable equipment $\quad 18+0.005$ points per peak watt

of renewable energy (or 0.01

points per peak watt of Renewable Energy if grid-connected)

Resource impacts

Resource efficient materials $\quad 70$

Reduce, reuse, recycle 23

Encourage waste reuse and recycling for homeowners 3

Waste efficiency

18

Occupant health/Indoor air quality

Minimize sources of pollutants 41

Provide ventilation to remove generated pollutants $\quad 12$

Occupant education and Operations and Maintenance 9 


\section{Southern Arizona Green Building Alliance (in progress)}

\section{Overview:}

Green Building Program is in its infancy and details are still being determined.

Certification method:

TBA

Levels of certification:

One-, two-, or three-armed "saguaro"

Qualification:

TBA

Year of inception:

TBA

Number of builders:

TBA

Incentives offered to builders to participate: TBA

Number of homes constructed to date:

TBA

Contact information:

Loretta Ishida

The Development Center for Appropriate Technology (DCAT)

PO BOx 27513

Tucson, AZ 85726-7513

p. $520-624-6628$

f. $520-798-3701$

Loretta@dcat.net

http://www.dcat.net

\section{Western North Carolina Green Building Council (in progress)}

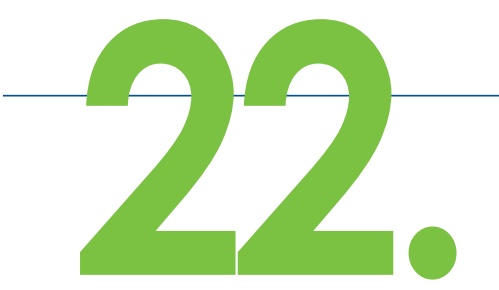

Overview:

No guidelines yet. The statewide program is in a very early stage of development. They are currently looking for funding to move the program to the draft stage. They are tentatively planning on teaming with the North Carolina Solar Center.

Certification method: TBA

Levels of certification:

TBA

Qualification:

TBA

Year of inception:

TBA

Number of builders:

TBA

Incentives offered to builders to participate:

TBA

Number of homes constructed to date:

TBA

Contact information:

Cindy Patton

PO Box 8427

Asheville, NC 28814

p. 828-251-5888

sheltereco@earthlink.net

http://www.main.nc.us/wncgbc 


\section{Alameda County (California) (in progress)}

\section{Overview:}

Under Development

Certification method:

TBA

Levels of certification:

TBA

Qualification:

TBA

Year of inception:

TBA

Number of builders:

TBA

Incentives offered to builders to participate:

TBA

Number of homes constructed to date:

TBA

Contact information:

Mari Soll

p. 510-614-1699

Chula Vista (California) GreenStar Building Incentive Program (in progress)

Overview:

Under Development

Certification method:

TBA

Levels of certification:

TBA

Qualification:

TBA

Year of inception:

TBA

Number of builders:

TBA

Incentives offered to builders to participate:

TBA

Number of homes constructed to date:

TBA

Contact information:

Mary Venables

p. 619-691-5296 


\section{Hudson Valley HBA Green Building Program (New York) (in progress)}

\section{Overview:}

Under Development

Certification method:

TBA

Levels of certification:

TBA

Qualification:

TBA

Year of inception:

TBA

Number of builders:

TBA

Incentives offered to builders to participate:

TBA

Number of homes constructed to date:

TBA

Contact information:

Jean Rowe

Hudson Valley Builders Association

338 Meadow Avenue

Newburgh, NY 12550

p. 845-562-0002

http://www.hvbuilder.com

Schenectady HBA Green Building Program (New York)

(in progress)

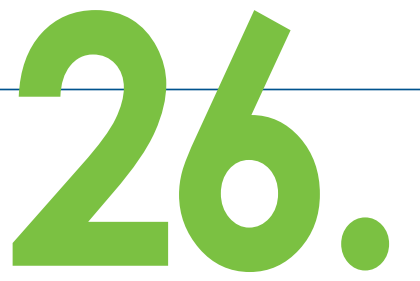

Overview:

Under Development

Certification method:

TBA

Levels of certification:

TBA

Qualification:

TBA

Year of inception:

TBA

Number of builders:

TBA

Incentives offered to builders to participate:

TBA

Number of homes constructed to date:

TBA

Contact information:

Rita Sickles

Schenectady Builders and Remodelers Association

1004 Princetown Road

Schenectady, NY 12306

p. 518-355-0055

http://www.schenectadybuilders.com 


\section{Conclusion}

For more information on Green Building Programs, contact the NAHB Research Center, 400 Prince George's Boulevard, Upper Marlboro, MD 20774-8731, (800) 638-8556, or visit our website at www.toolbase.org.

This document can also be downloaded from www.toolbase.org. 


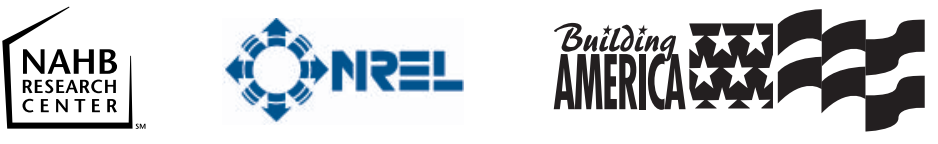

